\title{
Flexible Tube-Shaped Neural Probe for Recording and Optical Stimulation of Neurons at Arbitrary Depths
}

\author{
Shunsuke Tamaki*, Toshinobu Kuki ${ }^{1}$, Tadao Matsunaga², \\ Hajime Mushiake ${ }^{1}$, Yoshihito Furusawa ${ }^{3}$ and Yoichi Haga \\ Graduate School of Biomedical Engineering, Tohoku University, \\ 6-6 Aza Aoba, Aramaki, Aoba-ku, Sendai 980-8579, Japan \\ 'Department of Physiology, Graduate School of Medicine, Tohoku University, \\ 2-1 Seiryo-machi, Aoba-ku, Sendai 980-8575, Japan \\ ${ }^{2}$ Tohoku University Micro System Integration Center, \\ 6-6 Aoba, Aramaki, Aoba-ku, Sendai 980-8579, Japan \\ ${ }^{3}$ Department of Physical Medicine and Rehabilitation, Graduate School of Medicine, \\ Tohoku University, 2-1 Seiryo-machi, Aoba-ku, Sendai 980-8575, Japan
}

(Received December 15, 2014; accepted February 20, 2015)

Key words: tube-shaped neural probe, transparent, optical fiber, optical stimulation at arbitrary depth, nonplanar photofabrication

Transparent tube-shaped neural probes consisting of 8 recording areas on the surface of a cylinder and a movable optical fiber that is set in a groove of a stainless steel needle at the core have been developed. The recording areas are placed perpendicularly to the brain surface so that neural activities can be recorded across cortical layers. The movable metal needle with optical fiber enables optical stimulation of neurons surrounding the recording areas at any depth in the cortex. The neural probe was fabricated and examined for its applicability in optogenetic studies in neuroscience. The fabricated probe successfully recorded neural activities from the cortex of an anesthetized animal while optical stimulation was delivered successively at any depth of the cortex. Distinct neural activation patterns depending on the depth of optical stimulation were found. It was confirmed that the fabricated probe is suitable for optical manipulation for analysis of neural activities across cortical layers and simultaneously monitoring the effects of stimulation on neural activities.

\footnotetext{
*Corresponding author: e-mail: shunsuke.tamaki@bme.tohoku.ac.jp
} 


\section{Introduction}

Brain research is required for the application of a brain-machine interface (BMI). Various approaches and techniques are employed in brain research, one being the use of electrodes to record neural activities resulting from electrical transmission between neurons. Particularly for monitoring a neural circuit, it is indispensable to record multiple neural activities across a circuit simultaneously. For this purpose, various types of neural probe have been proposed. ${ }^{(1-3)}$

Neural probes with silicon and polymer substrates, such as parylene (polypara-xylylene) and polyimide-based neural probes, have been developed because microfabrication technology can be used for these materials. ${ }^{(4,5)}$ Owing to the flexibility of materials such as parylene, smooth insertion is difficult. A neural probe with a microfluidic channel filled with polyethylene glycol (PEG) to attain stiffness has been developed.(6) A three-dimensional (3D) sheath structure probe with a microwire for attaining stiffness has also been developed. ${ }^{(7)}$

The simultaneous selective stimulation and monitoring of neurons are required. To this end, newly developed neuroengineering techniques are required, called optogenetics, which enable cell-specific investigation of neural circuit functioning with optical stimulation (wavelength $=400-560 \mathrm{~nm}$ ) by targeted expression of channelrhodopsin-2 (ChR2). ${ }^{(8,9)}$ Therefore, various neural probes with optical illumination sites near the recording areas have been developed. Neural probes that have multiple recording areas and one or two optical fibers have been developed. ${ }^{(10)}$ These probes are fabricated by fixing optical fibers on commercially available neural probes. However, the limitation of the number of optical fibers in these probes is problematic in that the increase in the number of optical fibers increases the sectional area and requires complex fabrication. A probe with multiple waveguides has been developed.(11) It has 12 silicon oxynitride multimode waveguides coated with aluminum, and can deliver $33 \%$ of light from the optical fiber. $^{(11)}$ Also, recording probes with waveguides have been developed.(12-14) These probes have multiple recording areas and one or multiple waveguides. When these devices are employed, a high-intensity light source is required because the output power of the tip is diluted by the loss of input coupling. As noted above, the optical stimulation power and sites of these probes are limited.

In a previous work, a polyimide-based tube-shaped neural probe with one optical stimulation site using an optical fiber was proposed and developed.(15) The probe has a working channel. When it is inserted into the brain through the dura mater, it is stiff because a metal needle is set in the working channel. After insertion, the metal needle is removed from the working channel and the empty working channel is then used for the insertion of an optical fiber for stimulation. This probe has only one stimulation site although its stimulation power is sufficient to evoke neural activities. Insertion into the brain was not smooth, which may be caused by steps (about $30 \mu \mathrm{m}$ ) between the edges of a hole as the stimulation site with cutting of a part of the unclear polyimide tube and the tube surface.

To achieve high-power stimulation at arbitrary depths simultaneously with monitoring, a combination of an optically transparent tube-shaped neural probe with 
a smooth surface and one optical fiber was developed. The tube is made of parylene, which has about $80 \%$ light transmission at the ChR2-sensitive wavelength bands (wavelength $=400-560 \mathrm{~nm}$ ). ${ }^{(9,16)}$ Parylene is also a biocompatible material. ${ }^{(17)}$ Moreover, parylene coating of uniform thickness can be obtained. Multiple recording areas are set on the surface of the tube. An optical fiber with sideway illumination is used for stimulation. The optical fiber is set and fixed in the groove of a metal needle. A metal needle with an optical fiber is used owing to its sufficient stiffness for inserting into the brain. After insertion, moving of the metal needle with an optical fiber set in an optical transparent tube enables arbitrary-depth stimulation.

Artifacts of photoelectric effects emerge when the metal part is illuminated with light.(18) To prevent the formation of artifacts, the metal needle with an optical fiber set in the parylene tube is oriented toward a region without electrodes.

\section{Structure}

Figure 1 shows the concept of the proposed neural probe. The substrate of the probe is an optically transparent material made of parylene. The probe has a working channel, and when it is inserted into the brain, it is stiff because a stainless steel needle with an optical fiber is set in the working channel. After insertion, it is possible to stimulate neurons at arbitrary depths by moving the metal needle with an optical fiber and to record neural activities without moving of recording areas.

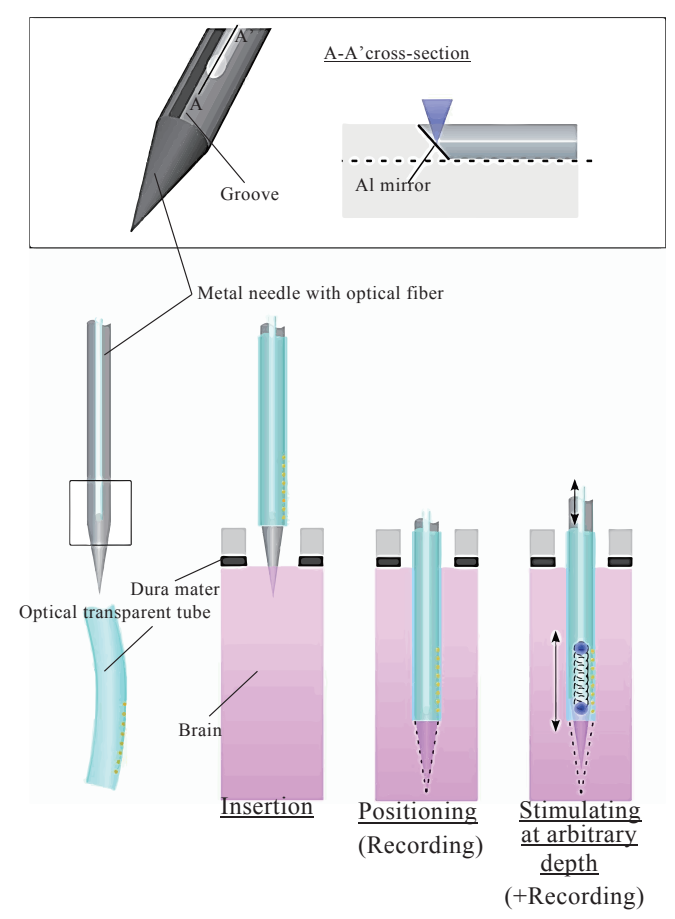

Fig. 1. (Color online) Concept of proposed neural probe. 
Figure 2(a) shows the structure of the proposed neural probe. The probe has $8 \mathrm{Au}$ recording areas $(\phi 25 \mu \mathrm{m})$ at $200 \mu \mathrm{m}$ intervals on a parylene tube (inner diameter, $330 \mu \mathrm{m}$; outer diameter, $360 \mu \mathrm{m}$ ) for recording the local field potential (LFP) of neural activities. The outer diameter of $360 \mu \mathrm{m}$ was based on the diameter of a commercially available neural probe (Plexon Inc., U-probe). The probe is composed of two layers for getting enough space to fabricate 8 recording areas and to increase recording areas in the future. Each layer has 4 recording areas. The illumination area is positioned at the parylene wall without metal recording areas or interconnections to prevent the photoelectric effect, as shown in Fig. 2(b).

The stainless steel needle is used to attain stiffness and has a groove for placement of an optical fiber with a diameter of $300 \mu \mathrm{m}$. The multimode silica optical fiber (core diameter, $50 \mu \mathrm{m}$; cladding diameter, $125 \mu \mathrm{m}$ ) is cut at an angle of $45^{\circ}$ and $\mathrm{Al} / \mathrm{Cr}$ is sputtered on the cut section for stimulation in the vertical direction, as shown in Figs. 1 and 2. For the insertion of and moving of the needle with an optical fiber inside the tube, the diameter of the tube is larger than $300 \mu \mathrm{m}$.

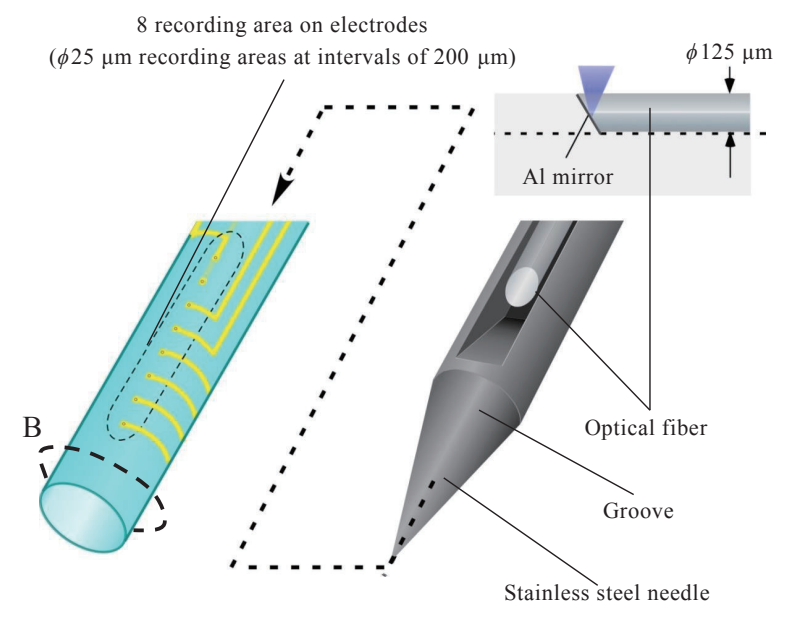

(a)

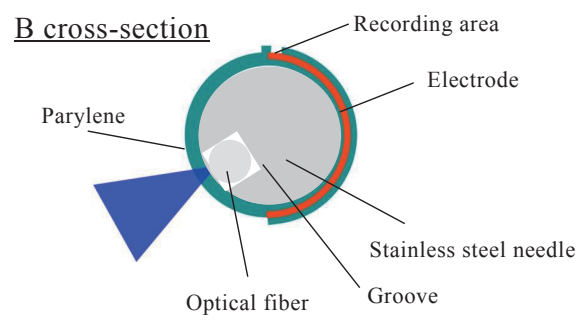

(b)

Fig. 2. (Color online) Tip design of proposed neural probe. (a) Parylene tube and metal needle with optical fiber and (b) metal needle with optical fiber set in the tube. 
The probe is attached to a handling jig to enable connection to the connector (Omnetics Connector Corp., CON/8o50m-10P) and attached to "jig 1". The stainless steel needle with optical fiber is attached to "jig 2", which is connected to the tip of the nonrotating spindle-type micrometer head (Mitsutoyo Co., MHK-15). Only the stainless steel needle with an optical fiber that is connected to "jig 2" is moved by rotating the micrometer head (Fig. 3). This allows stimulation at arbitrary depths of the brain without changing recording areas.

\section{Fabrication}

The fabrication process of the tube-shaped probe is shown in Fig. 4. The recording electrodes of the proposed probe are photofabricated by a nonplanar photofabrication process on a cylindrical surface. ${ }^{(19,20)}$ The metal part is made of $\mathrm{Au}$, and the insulator is coated with parylene (Kisco Ltd., Dix-SR).

A stainless steel rod was used as the base material. Photoresist as a sacrificical layer $(15 \mu \mathrm{m})$ was prepared by electrodeposition (Honny Chemical Co., AP-1000) and parylene $(5 \mu \mathrm{m})$ was applied to the rod by chemical vapor deposition (CVD). $\mathrm{Au} / \mathrm{Cr}$ as the seed layer for electroplating was sputtered on the parylene substrate by rotating of the sample. Positive photoresist $(10 \mu \mathrm{m})$ was prepared using a spray coater. Recording electrodes were patterned using a nonplanar exposure system, which consists of a UV spot laser and computer-controlled multiaxial stages (Fig. 4). After the exposed areas had been electroplated with $\mathrm{Au}(3 \mu \mathrm{m})$, the photoresist and seed layer were removed by wet etching. Parylene was coated $(5 \mu \mathrm{m})$ for insulation. After a second layer had been fabricated by the same process, parylene on the recording areas for recording and electrical pads were removed by laser ablation using a Nd:YAG laser (wavelength $=355$

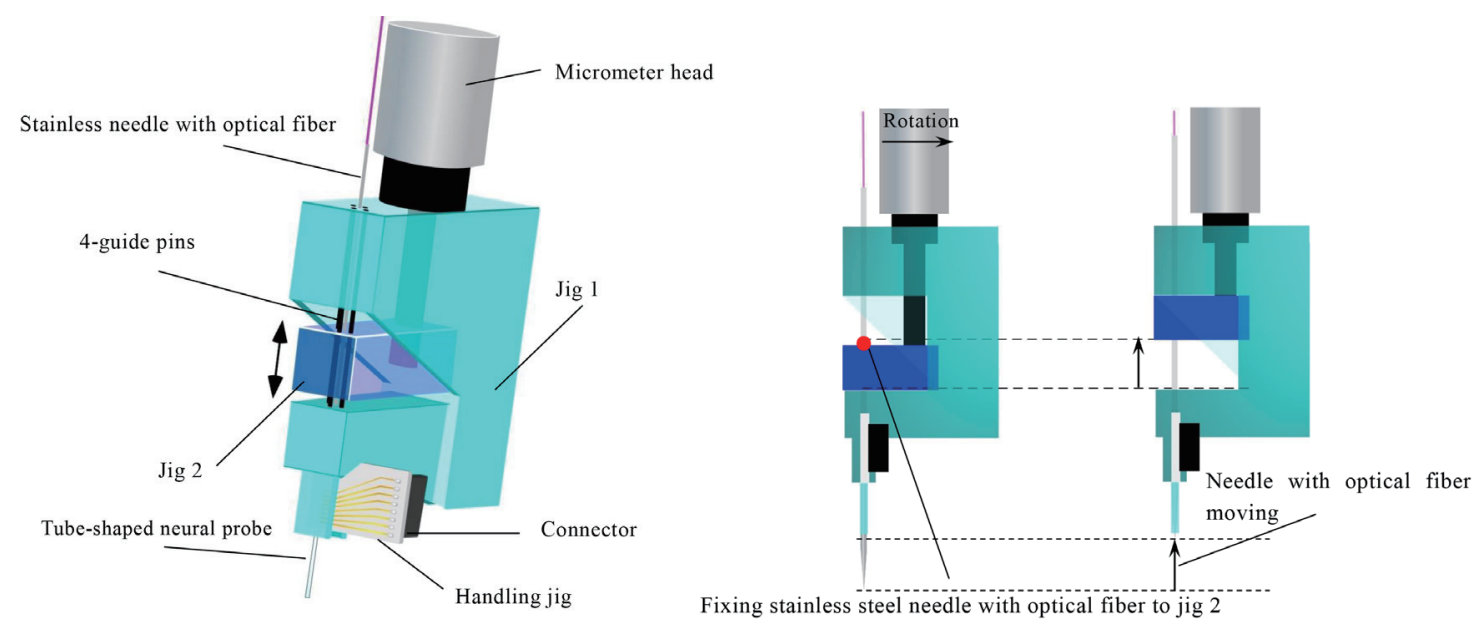

Fig. 3. (Color online) Overview of proposed neural tube. 


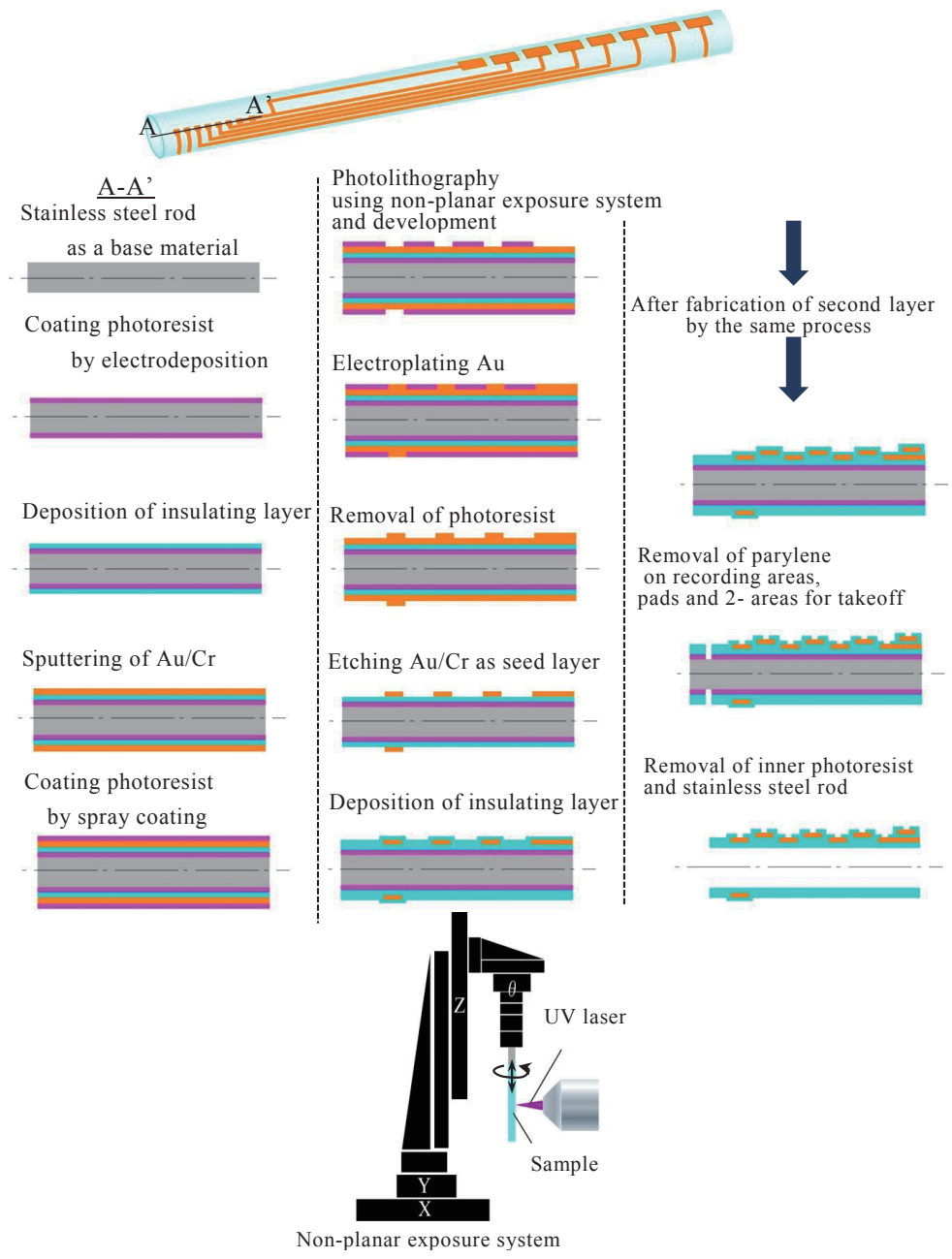

Fig. 4. (Color online) Process flow for tube-shaped probe.

$\mathrm{nm}$ ) system that had multiaxial stages. The fabricated probe was attached to a handling jig, which has interconnections and a connector, and electric connection between the electrical pads on the probe and the interconnections on a handling jig was formed using a conductive adhesive (Epoxy Technology Inc., EPO-TEK H20E).

For optical stimulation, an optical fiber with a mirror was fabricated by the lift-off process. An optical fiber was attached to the grooved glass plate by a photoresist and cut off at a $45^{\circ}$ angle using a dicing saw machine. After cutting, $\mathrm{Al} / \mathrm{Cr}(\mathrm{Al}=200 \mathrm{~nm}, \mathrm{Cr}=$ $70 \mathrm{~nm}$ ) to form a mirror was sputtered on the optical fiber attached to the grooved glass plate. The $\mathrm{Al} / \mathrm{Cr}$ part excluding that of the cut section was removed by lift-off. 
"Jig 1" and "jig 2" were prepared using a 3D printer (Keyence Co., Agilista-3000). The handling jig with the probe was attached to jig 1, and the stainless needle with the optical fiber was attached to jig 2 after the stainless steel needle had been set in the probe.

Figure 5(a) shows a successfully fabricated probe with 8 Au recording areas (diameter $25 \mu \mathrm{m})$ at $200 \mu \mathrm{m}$ intervals on a cylindrical surface. An overview of the fabricated neural probe is shown in Fig. 5(b).

Figure 6(a) shows the cut section of the optical fiber onto which $\mathrm{Au} / \mathrm{Cr}$ was sputtered. The fabricated optical fiber was set in the groove of the stainless steel needle and illuminated by connecting to the laser diode (wavelength $=490 \mathrm{~nm}$ ), as shown in Fig. $6(\mathrm{~b})$. The tip of the optical fiber was confirmed to be moved by rotating the micrometer head. The metal needle was set in the parylene tube with optical illumination at arbitrary depths by the optical fiber connected to the laser diode (wavelength $=490 \mathrm{~nm}$ ) as shown in Fig. 6(c).

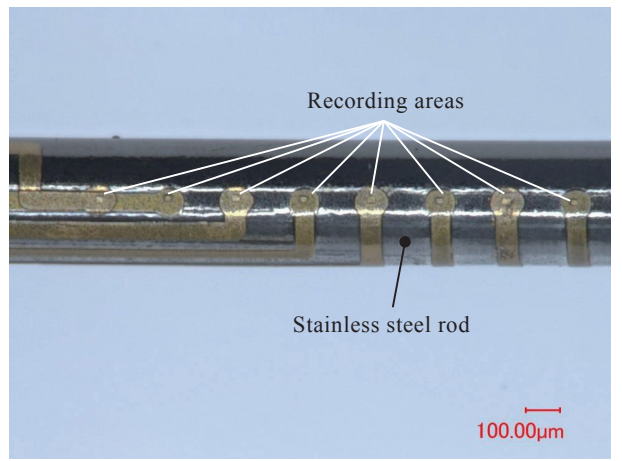

(a)

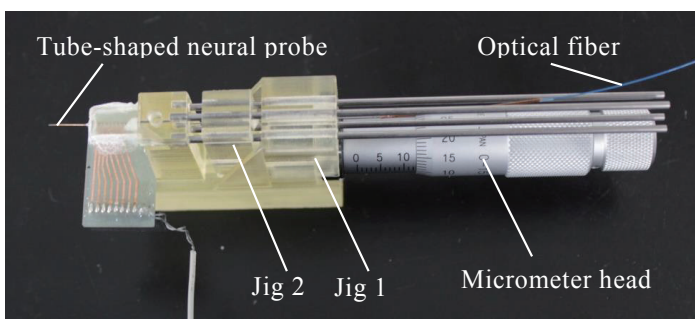

(b)

Fig. 5. (Color online) Fabricated probe. (a) Tip of fabricated probe and (b) overview of neural probe.
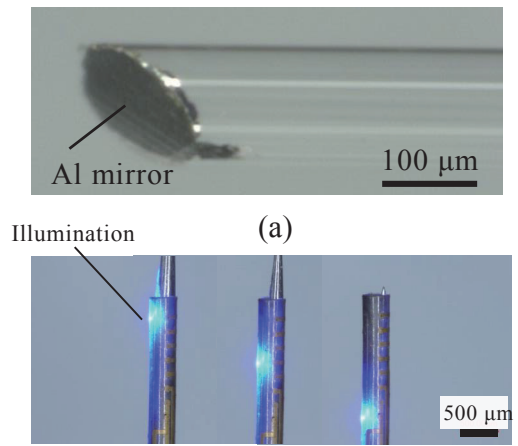

(c)

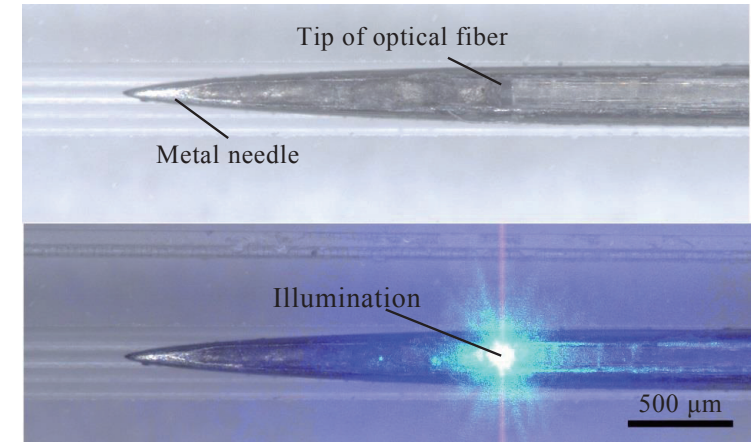

(b)

Fig. 6. (Color online) Fabricated metal needle with optical fiber. (a) Fabricated optical fiber with mirror, (b) fabricated stainless needle with optical fiber, and (c) illumination at arbitrary depths. 


\section{Evaluation and Results}

\subsection{Impedance test}

The fabricated probe had 8 recording areas, and the impedance magnitude of the respective recording areas in saline was measured with an LCR meter (Hioki E.E. Corporation, LCR meter) from 0.1 to $10 \mathrm{kHz}$, which includes the frequencies of neural activities. $\mathrm{An} \mathrm{Ag} / \mathrm{AgCl}$ electrode was used as the reference electrode and a Pt electrode was used as the counter electrode.

The impedance magnitude of each of the 8 recording areas at frequencies from 0.1 to $10 \mathrm{kHz}$ is shown in Fig. 7. The average impedance magnitude of all the recording areas at $1 \mathrm{kHz}$ was $678 \pm 110 \mathrm{k} \Omega$ (mean $\pm \mathrm{SD}, n=8$ ). This value is equal to those of conventional neural probes, such as axial array microelectrodes (FHC Corporate and Manufacturing). The relationship between recording area and electrolyte is represented by Randles circuit. The impedance magnitude decrease is caused by the capacitive component between the recording area and the electrolyte decreasing with increase of frequency. ${ }^{(21)}$

\subsection{Evaluation of in vivo neural recording and stimulation}

PEG was applied to temporarily attach the tip of the fabricated probe to the stainless steel needle with the optical fiber to form a smooth surface between the tip of the tube and the needle (Fig. 8). After inserting the probe into the brain, PEG was dissolved by flowing saline solution over the surface of the parylene tube using a syringe. Dissolution of PEG was determined if the needle setting in the tube could be moved.

The stimulation points are shown in Fig. 9. At the respective points, neural activities were measured before and after stimulation. All experimental procedures were approved by the Tohoku University Committee for Animal Research.
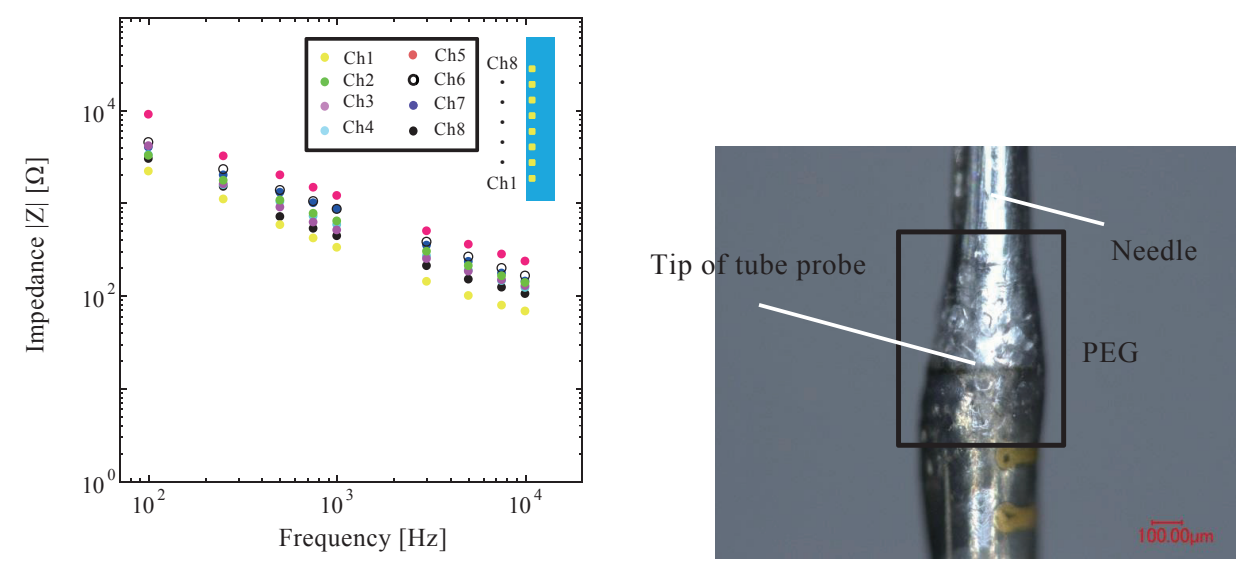

Fig. 7 (left). (Color online) Impedance of fabricated probe.

Fig. 8 (right). (Color online) PEG is applied at the tip of the fabricated probe. 


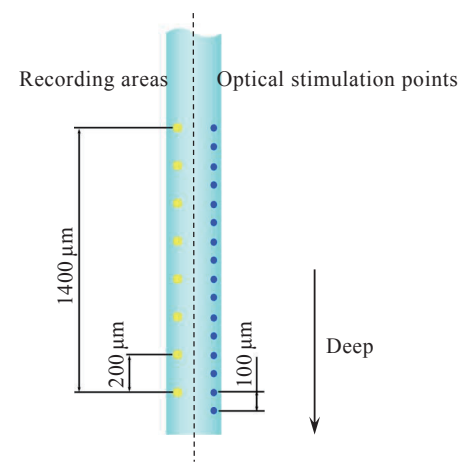

Fig. 9. (Color online) Stimulation points in this experiment.

One adult Wistar rat expressing ChR2 was used. The rat was anesthetized with ketamine hydrochloride $(100 \mathrm{mg} / \mathrm{kg})$ and xylazine $(10 \mathrm{mg} / \mathrm{kg})$. All the surgical or pressure points were injected with $0.25 \%$ lidocaine hydrochloride. Surgical anesthesia was determined by the absence of the vibrissa motor response, leg withdrawal response, and eye-blink reflex. Additional doses of the ketamine and xylazine mixture were given through an intraperitoneal catheter as needed. The head of the rat was fixed by a head fixtator. Its cranial bone around the somatosensory cortex $(2.5 \mathrm{~mm}$ lateral and $2.5 \mathrm{~mm}$ caudal from the bregma) and dura mater were removed and a bone screw for grounding was set on the cerebellum with reference to a brain atlas. ${ }^{(22)}$

The probe was inserted into the brain using a manipulator and the bone screw was attached to the connector as the ground. The measured neural signals were amplified and processed using a data logging system (Plexon, Omniplex).

First, neural activities including action potential and LFP were measured without external stimulation. Second, optical stimuli at arbitrary depths were applied by the stainless steel needle with an optical fiber set in the tube [Fig. 6(c)]. The metal needle with optical fiber was connected to the laser diode $(490 \mathrm{~nm})$ and the light output was adjusted to $90 \mu \mathrm{W}$ at the tip of the optical fiber set in the tube. Light pulses of $2 \mathrm{~ms}$ were applied at $2 \mathrm{~Hz}$ for optical stimulation. The neural activities resulting from optical stimulation were measured.

LFP data obtained from the fabricated probe were examined by current source density (CSD) analysis. Equation (1) was previously used for filtering high-frequency noise. ${ }^{(23)}$

$$
\overline{f_{n}}=0.23 f_{n-1}(t)+0.54 f_{n}(t)+0.23 f_{n+1}(t)
$$

where $f_{n}(t)$ is the position of the recording area of the neural probe.

Equation (2) was then used for CSD. ${ }^{(24,25)}$

$$
\bar{I}_{n}(t)=-\left\{\frac{\bar{f}_{n-1}-2 \bar{f}_{n}(t)+\bar{f}_{n+1}(t)}{h^{2}}\right\}
$$

To obtain mean CSD and LFP, 40 data obtained $40 \mathrm{~ms}$ before and after the simulation were averaged. 


\subsection{Results of in vivo neural recording and stimulation}

\subsubsection{The fabricated probe was successfully inserted into the brain}

To ascertain the successful insertion of the probe, the recorded LFPs and CSDs calculated from LFPs were examined (Fig. 10). CSD analysis revealed the LFP current source by removing volume conduction (electrical leakage via cerebrospinal fluid). Although the Ch5 signal was not properly measured, all the other signals were successfully measured. Recorded signals consisted of a fluctuating active up-state and a flat quiet down-state. They alternated at about $1 \mathrm{~Hz}$ (Fig. 10). This is a characteristic of slow oscillation caused by ketamine-xylazine induced anesthesia. ${ }^{(25)}$ Therefore, insertion of the probe into the brain was successful.

\subsubsection{Examples of stimulation-depth-dependent response: deep-layer stimulation evoked strong potentials followed by propagation of potentials to the upper layer}

To understand the different responses depending on stimulation depth roughly, examples of averaged LFPs and CSDs in response to upper-layer stimulation [Fig. 11(a)] and to deep-layer stimulation are shown in Fig. 11(b). CSDs were calculated with the exclusion of Ch5. The CSD of Ch4 was calculated by using Ch2 and Ch6, and the CSD of $\mathrm{Ch} 6$ was calculated by $\mathrm{Ch} 4$ and $\mathrm{Ch} 8$, respectively. The CSD of Ch5 was set at zero.

Upper stimuli resulted in weak evoked potentials near the upper layers [Fig. 11(a)]. On the other hand, deep-layer stimulation resulted in the strong evoked potentials in the deep layers, followed by evoked potentials in the upper layers. Therefore, these results show that each depth stimulation evoked potentials in each layer, whereas only deep-layer stimulation caused propagated potentials in the upper layers after deep-layer evoked potentials. No artifacts of the photoelectric effect emerged in these results.

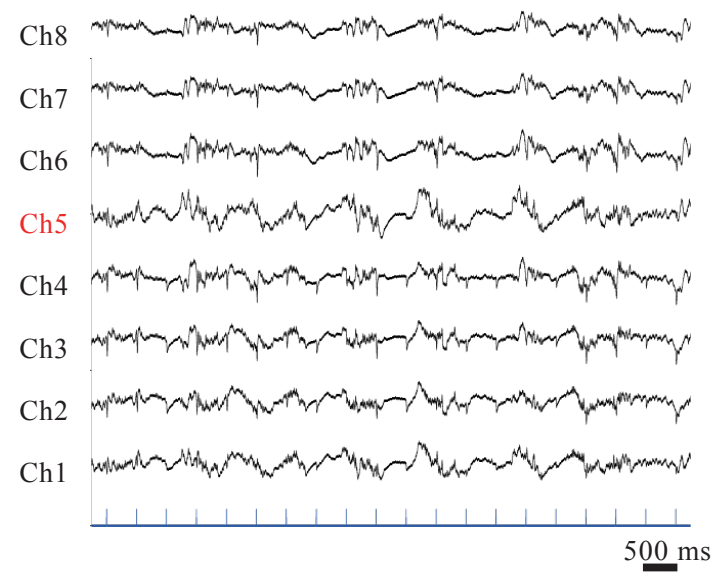

Fig. 10. (Color online) Signals obtained from recording areas (slow oscilation). 

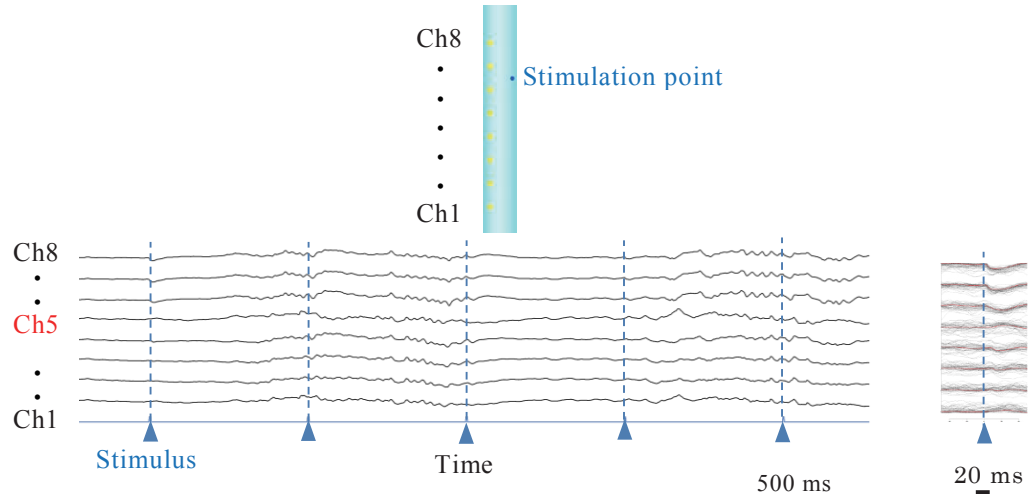

$20 \mathrm{~ms}$
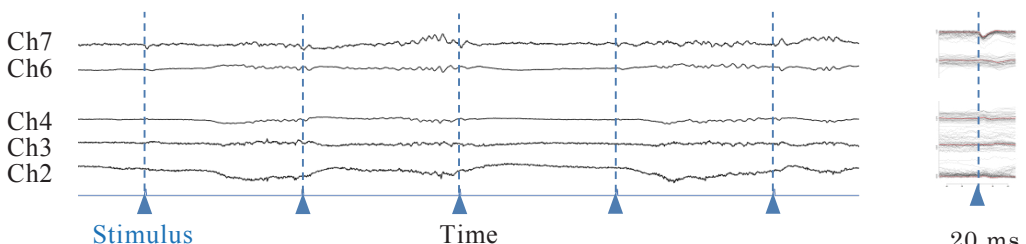

(a)

$500 \mathrm{~ms}$
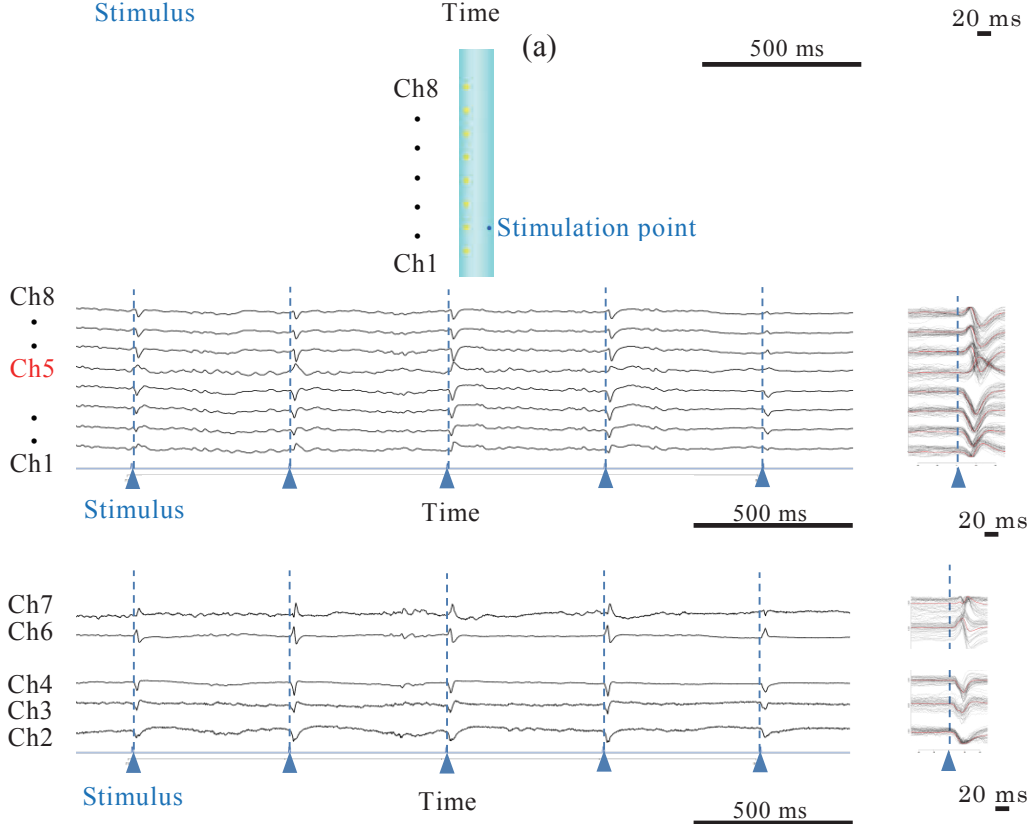

(b)

Fig. 11. (Color online) LFP and CSD responses (a) to upper-layer stimulation and (b) to deep-layer stimulation. 


\subsubsection{Systematic stimulation-depth-dependent response: layer 5 stimulation evoked strong sink responses propagating to the upper layer}

To show the dependence of different evoked potentials on stimulation depth, peristimulation averaged CSDs at all stimulation depths are shown in Fig. 12. The horizontal direction indicates time and the vertical direction indicates the CSD of respective recording areas. The CSDs of Fig. 12 were calculated $40 \mathrm{~ms}$ before and the after the simulation, and the color range is set between max sink and source from -40 to $40 \mathrm{~ms}$. From deep stimulation (a) to upper stimulation (p), mean CSDs were sorted. Red indicates the sink, which means excitatory activity in terms of CSD. Strong sink responses after stimulation were concentrated within stimulation depths e to g, not within all deep layers. Depths from e to $g$ were assumed to correspond to cortical layer 5. ${ }^{(22)}$ These results show that stimulation at around layer 5 caused stronger evoked potentials than stimulation of any other layers.

To determine the dependence of different propagated potentials on stimulation depth, CSDs are calculated in $10 \mathrm{~ms}$ intervals after stimuli. Figure 13 shows the sink matrix (horizontal stimulation depth vs perpendicular recording depth). CSDs [Fig. 13(b) upper left] were calculated $10 \mathrm{~ms}$ after the stimulation, and the color range is set between max sink and source from 0 to $10 \mathrm{~ms}$. Source responses are not displayed in Fig. 13. Initially, early evoked sink responses appeared around the stimulation depth [Fig. 13(b)

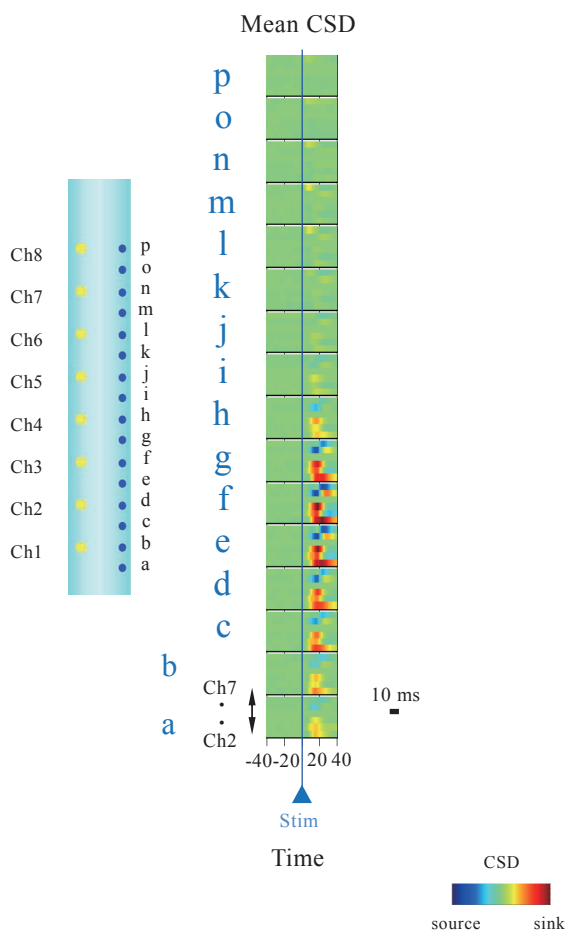

Fig. 12. (Color online) Peristimulation averaged CSDs at various depths. 


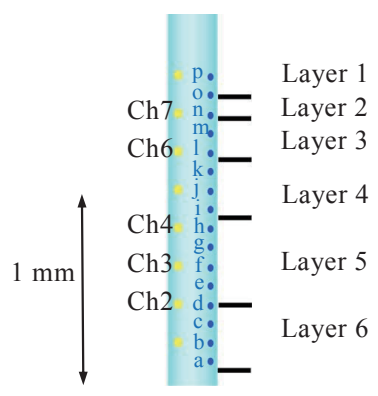

(a)
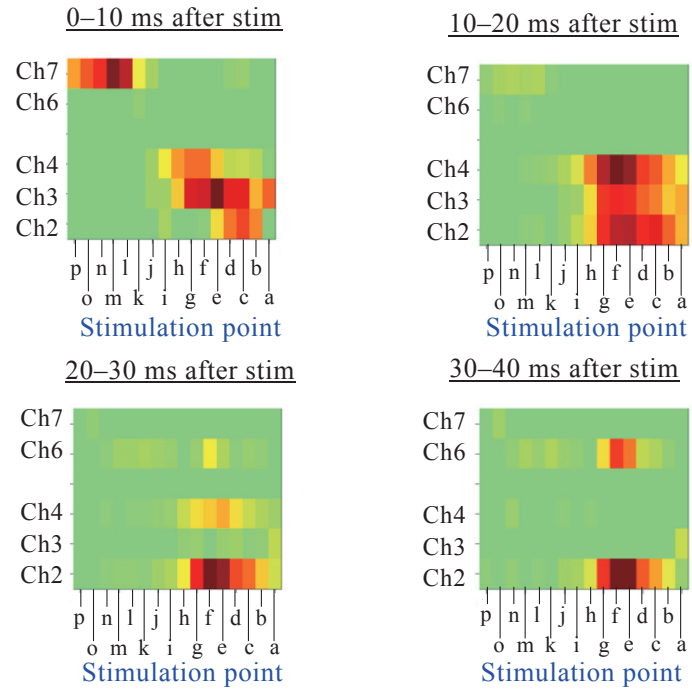

$\underline{30-40 \mathrm{~ms} \text { after stim }}$

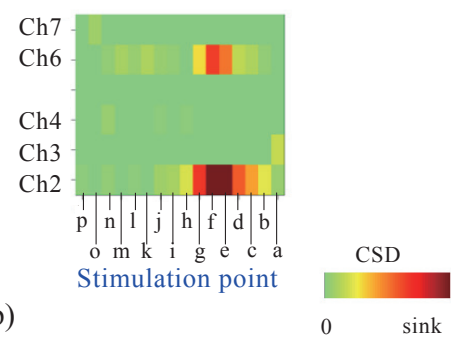

Fig. 13. (Color online) Induced potential and potential propagation distribution for respective depth stimulation (a) Schematic representation of in vivo experiment and (b) induced potential and potential propagation distribution for respective depth at $0-10,10-20,20-30$, and 30-40 ms after stimulation.

upper left]. Deep stimulation (a-i) induced sink in the deep layer (Ch2-Ch4) and upper stimulation (1-p) induced sink responses in the upper layer (Ch7). In Fig. 13(b) upper left, max red color shows very weak sink responses directly evoked by opto-stimulus in each layer by color range setting max sink from 0-10 ms although the CSDs look green in Fig. 12. Strong propagated sink responses appeared around some parts of the stimulated deep layers [Fig. 13(b) lower right, from e to g]. These stimulation points induced the sink responses, not only in the deep layers at $\mathrm{Ch} 2$, but also in the upper layers at Ch6. The stimulation points from e to $g$ are assumed to be around layer 5.(21) Therefore, these results indicate that stimulation of layer 5 propagated to upper layers.

The results show that stimulation of layer 5 evoked strong sink responses, which propagated to the upper layers, whereas stimuli to the other layers evoked weak sink responses, which did not propagate to the other layers. 


\section{Discussion}

The successfully fabricated probe has sufficient impedance for recording neural activities. Results of the in vivo experiment showed that the fabricated probe could be used for optical stimulation at arbitrary depths and to record different responses of LFPs and CSDs along cortical layers, depending on stimulation depth. Results of this study suggest that this probe meets the needs of neuroscientists: strong, comprehensive stimulation of each cortical layer sufficient to cause layer-specific responses in vivo.

The fabricated probe was successfully inserted into the brain. For reduction of the risks of infection and bleeding of research animals caused by the peeling of the dura mater, the insertability of the probe into the dura mater will be confirmed in the future. Only one recording area was not measured properly, whereas recording of the other areas showed slow oscillations across layers under ketamine-xylazine induced anesthesia (Fig. 10). (26) Failure in one recording area was probably caused by insulation peeling, perhaps due to the impact of setting to the manipulator or bumping into something during the setup of the in vivo experiment. This indicates that careful handling of the probe is necessary.

The stimulation of the upper part of the deep layers, probably layer 5, evoked strong sink responses followed by propagation of the responses to the upper layers (Fig. 13). Layer 5 is indispensable for the up-state in slow oscillations. The activity of layer 5 , but not that of layer $2 / 3$, propagates to layer $2 / 3$ in slow oscillation. ${ }^{(27)}$ However, the propagations from layers 6,4 , and 1 to other layers in slow oscillation were not investigated at the causality level. Layer 5 , along with layer 6 , is often classified as a deep layer. The results suggest that layer 5 , but not layer 6 , is the activity-propagation source in the deep layers. The reason only stimulation of layer 5 caused strong potentials and propagation to other layers under ketamine anesthesia is expected as follows. Ketamine serves as an antagonist of glutamatergic $N$-methyl-D-aspartic acid (NMDA) receptors and exerts its anesthetic effect by acting on NMDA receptors. ${ }^{(28)}$ NMDA receptors are one of the receptors that mediate excitatory neural communication. According to developmental study of the effect of NMDA receptor antagonists on the neocortex, neocortical layer $2 / 3$ is functionally dependent on NMDA receptors at all ages, whereas neocortical layer $4 / 5 / 6$ is less dependent on NMDA receptors in adulthood. ${ }^{(29)}$ Ketamine anesthesia might inhibit intra-laminar neuronal communication triggered by optical stimulation only in layer $2 / 3$, because all the animals used in our study are adult rats. Optical stimulation could trigger intra-laminar neuronal excitatory communication in layers 4, 5, and 6. However, a question arises why stimulation of layer 5 selectively causes strong potentials and propagation to other layers. According to detailed review of cortical microcircuits, dense intra-laminar excitatory recurrent circuits are limited within layers $2 / 3$ and $5 .^{(30)}$ On the basis of these studies, we propose a plausible model to explain our findings in Fig 14. When optical stimulation is delivered across cortical layers, direct responses are evoked in each layer. Then, these weak evoked potentials would be amplified through recurrent circuits in layers $2 / 3$ and 5 . However layer $2 / 3$ is more dependent on NMDA receptors than layer 5, so evoked potentials became stronger in layer 5 than in layer $2 / 3$ by ketamine block of NMDA receptors. Without adequate 

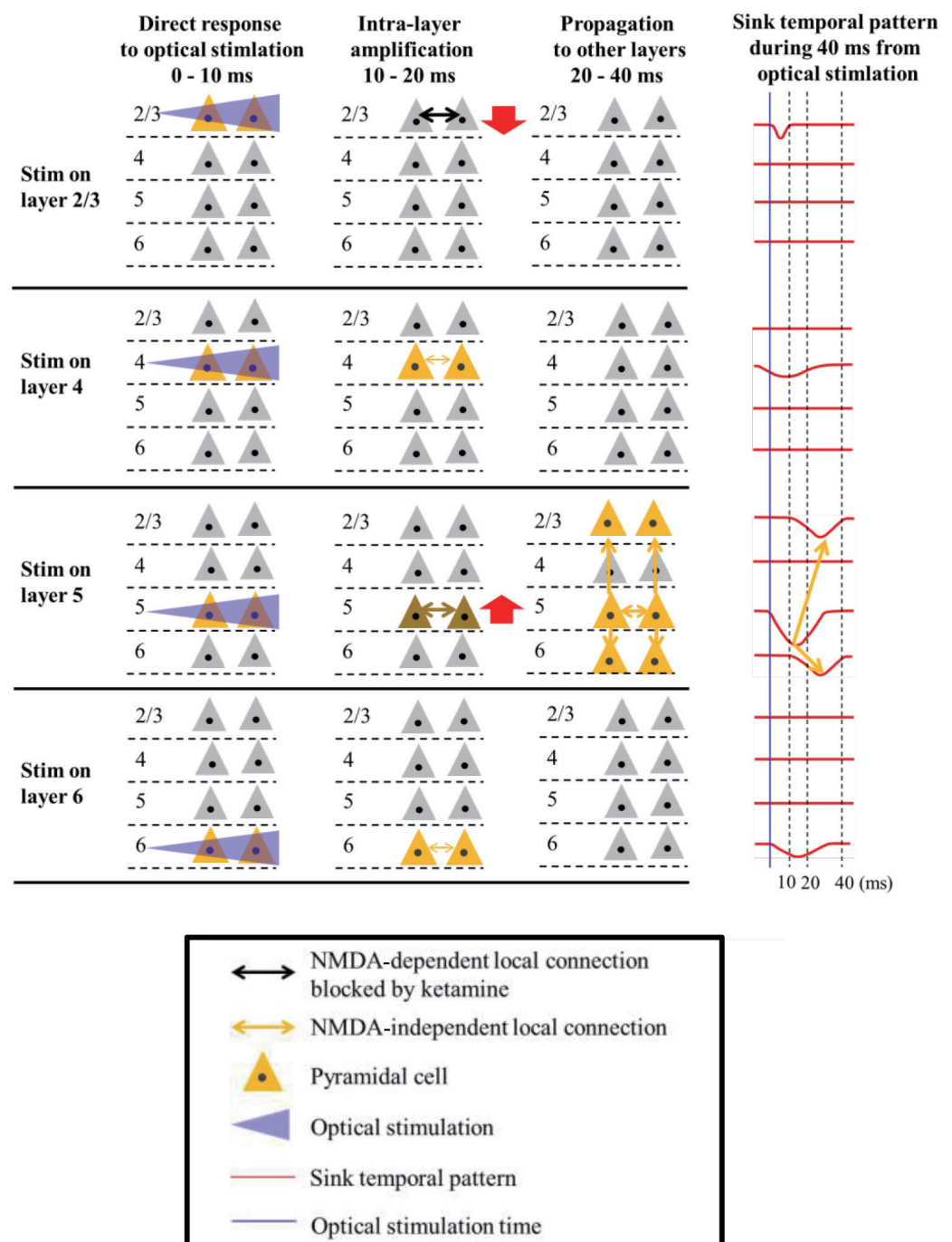

Fig. 14. (Color online) Expectation of neural responses to stimulation at each layer.

amplification through recurrent circuits, potentials in layers $2 / 3,4$, and 6 could not propagate to other layers, whereas layer 5 might be able to amplify their activity so that potentials from layer 5 could cause propagation to layers $2 / 3$ and 6 .

In short, the fabricated probe can stimulate at arbitrary depths sufficient for observing distinguishable functions corresponding to each layer. 
In neuroscience research, this probe will enable greater stimulation power and stimulation of more sites than conventional probes. ${ }^{(10,12-14)}$ Power-limited stimulation may not affect neural circuit function. Studies with limitation of the number of stimulations may not result in a comprehensive study across all layers. Without these limits, neuroscientists will be able to find neural circuit functions corresponding to each layer, even to each sublayer. Therefore, the proposed probe can be used for more detailed studies in neuroscience.

By decreasing the outer diameter of the neural probe, reduction of damage during its insertion into the brain is expected. Moreover, it is applicable in small animals such as mice. To do so, it requires consideration of both decreasing the diameter of the parylene tube and downsizing the electrode pattern design.

Decreasing the diameter of the tube is possible by using a base stainless steel rod with a smaller diameter during fabrication. It is possible to decrease the diameter to 290 $\mu \mathrm{m}$ without changing the current electrode pattern design. Further decrease in the outer diameter is possible by decreasing the width of the electrode pattern.

\section{Conclusions}

A transparent tube-shaped probe capable of stimulation at arbitrary depths was designed, fabricated, and evaluated. The fabricated probe has adequate impedances. The results of in vivo evaluation, confirmed that the fabricated probe can be inserted into the brain of a rat in which the dura mater has been peeled off. Neural activities were successfully measured except in one recording area. It was confirmed that light evoked-LFP changes were obtained from recording areas. After CSD analysis, sink (positive charges enter the neurons) responses from recording areas were observed near the optically stimulated area. The transparent tube-shaped probe enabled comprehensive stimulations and simultaneous recording of neural activities across the cortical layer. The transparent tube-shaped probe is indicated to be a useful tool for brain analysis.

\section{References}

1 K. D. Wise, J. B. Angell and A. Starr: IEEE Trans. Biomed. Eng. 3 (1970) 238.

2 J. S. Barna, J. C. Arezzo and H. G. Vaughan Jr.: Electroencephalogr. Clin. Neurophysiol. 52 (1981) 494.

3 T. Jellema, and J. A. W. M. Weijnen: J. Neurosci. Meth. 40 (1991) 203.

4 Y. Y. Chen, H. Y. Lai, S. H. Lin, C. W. Cho, W. H. Chao, C. H Liao, S. Tsang, Y. F. Chen and S. Y. Lin: J. Neurosci. Meth. 182 (2009) 6.

5 S. Takeuchi, T. Suzuki and K. Mabuchi: J. Micromech. Microeng. 14 (2004) 104.

6 S. Takeuchi, D. Ziegler, Y. Yoshida, K. Mabuchi and T. Suzuki: Lab Chip 5 (2005) 519.

7 B. J. Kim, J. T. W. Kuo, S. A.Hara, C. D. Lee, L. Yu, C.A. Gutierrez, T. Q. Hoang, V. Pikov and E. Meng: Lab Chip 13 (2013) 554.

8 A. M. Aravanis, L. P. Wang, F. Zhang, L. A. Meltzer, M. Z. Mogri, M. B.Schneider and K. Deisseroth: J. Neural Eng. 4 (2007) 143.

9 H. Wang, Y. Sugiyama, T. Hikima, E. Sugano, H. Tomita, T. Takahashi, T. Ishizuka and H. Yawo: J. Biol. Chem. 284 (2009) 5685. 
10 S. Royer, B. V. Zemelman, M. Barbic, A. Losonczy, G. Buzsaki and C. Mgee: Eur. J. Neurosci. 31 (2010) 2279.

11 A. N. Zorzos, E. S. Boyden and C. G. Fonstad: Opt. Lett. 35 (2010) 4133.

12 I.-J. Cho, W. B. Hyoung and E. Yoon: MEMS, 2010 IEEE 23rd International Conference on. IEEE (2010) pp. 995-998.

13 F. Wu, E. Stark, M.Im, I. J. Cho, E. S. Yonn, G. Buzsáki, K. D. Wise and E. Yoon: J. Neural Eng. 10 (2013) 056012.

14 S. Kanno, S. Lee, T. Harashima, T. Kuki, H. Kino, H. Mushiake, H. Yao and T. Tanaka: EMBC, 2013 35th Annual International Conference of the IEEE (2013) pp. 253-256.

15 S. Tamaki, T. Matsunaga, T. Kuki, Y. Furusawa, H. Mushiake and Y. Haga: Solid-State Sensors, Actuators and Microsystems, 2013 Transducers \& Eurosensors XXVII: The 17th International Conference on IEEE (2013) pp. 864-867.

16 D. Feili, M. Schuettler, T. Doerge, S. Kammer, K. P. Hoffmann and T. Stieglitz: J. Micromech. Microeng. 16 (2006) 1555.

17 E. M. Schmidt, J. S. Mcitosh and M. J. Bak: MBEC 26 (1988) 96.

18 X. Han, X. Qian, J. G. Bernstein, H. H. Zhou, G. T. Franzesi, P. Stern, R. T. Bronson, A. M. Graybiel, R. Desimone and E. S. Boyden: Neuron 62 (2009) 191.

19 S. Goto, T. Matsunaga, J. J. Chen, W. Makishi and Y. Haga: Proc. Microtechnologies in Medicine and Biology, 2006 International Conference on IEEE (2006) pp. 217-220.

20 T. Matsunga, R. Hino, W. Makishi, M. Esashi and Y. Haga: IEEJ Trans. Sens. Micromachines 129 (2009) 399.

21 Y. Kato, K. Maki, S. Furukawa and M. Kashino: Trans. Jpn. Soc. Med. Biol. Eng. 46 (2008) 522 (in Japanese).

22 G. Paxinos and C. Watson: The Rat Brain in Stereotaxic Coordinate (Vol. 6) (Academic press, New York, 2009).

23 P. Rapperlsberger, H. Pockberger and H. Petsche: Pflügers Archiv 389 (1981) 159.

24 J. A. Freeman and C. Nicholson: J. Neurophysiol. 38 (1975) 369.

25 S. Chauvettel, M. Volgushev and I. Timofeev: Cereb. Cortex 20 (2010) 2660.

26 M. Steriade, D. A. McCormick and T. J. Sejnowski: Science 262 (1993) 679.

27 R. Beltramo, G. D’Urso, M. D. Maschio, P. Farisello, S. Bovetti, Y. Clovis, G. Lassi, V. Tucci, D. D. P. Tonelli and T. Fellin: Nat. Neurosci. 16 (2013) 227.

28 T. Yamamura, K. Harada, A. Okamura and O. Kemmotsu: Anesthesiology 72 (1990) 704.

29 K. Fox, H. Sato and N. Daw: J. Neurosci. 9 (1989) 2443.

30 R. J. Douglas and K. A. Martin: Annu. Rev. Neurosci. 27 (2004) 419. 